\title{
Is surgical mystique a myth and double standard the reality?
}

L A Michel, P Johnson

J Med Ethics: Medical Humanities 2002;28:66-70

Clinically relevant attitudes and guidelines issued by a rational evidence based medicine (EBM) approach, integrate individual clinical expertise with the best available external clinical evidence from systematic research. Many surgeons, however, while considering the ultraliberal world they are practising in, and fearing that the primary goal of managed care in a market environment is reducing cost in order to make profit or decrease spending, remain suspicious of this kind of tentative protocol driven medicine when applied to surgical practice. If surgeons want to develop a health policy agenda that emphasises patient care issues above providers' or payers' interests, they should also enhance education programmes, improve continuing objective assessment of the way surgery is performed, face moral issues raised by innovation, and assume an increased leadership role in sound critical evaluation of non-validated new techniques. They should no longer consider EBM as a weapon turned against the surgical profession, but rather see it as a tool that may provide some answers to chronically unresolved questions in the evolving art of surgery.

See end of article for authors' affiliations ...........................

Professor L A Michel, Surgical Services, Mont-Godinne University Hospital, Yvoir, B-5530, Belgium;

michel@chir.ucl.ac.be

Accepted for publication 12 August 2002
T o what extent is current surgical practice, taken as a whole, actually supported by satisfactory scientific evidence? The idea that clinical practice should be rigorously based on the best scientific evidence is not new. ${ }^{1}$ Pressure to contain the inflationary growth of health care costs, however, and what is often felt by surgeons to be a focus on their high cost specialty, has been increasing over the past few decades. Until relatively recently, attempts to restrain this growth had only minimal or transient effects and the failure to control costs has led to the current climate, one that attempts to control unit price and question the effectiveness of care. In such a climate, cost effectiveness, cost benefit, cost analysis, and evidence based medicine are sometimes only buzzwords that disguise the slipperiness of key concepts that bedevil clinical decision making.

Early attempts to control health care costs were unsuccessful, in large part, because it was believed that the relationship between cost and quality was strictly positive. In such a model, reducing health care expenditures would only reduce quality. Defining quality in health care was (and still is), however, somewhat elusive. Health care providers, including surgeons and hospitals, claimed high quality, yet similar claims were accompanied by significant variations in actual cost. Health care payers then reasoned that if the providers claimed equal quality, they would simply practise good business and purchase quality from the least expensive providers. As a consequence, the current belief of health care payers is that the relationship between quality and cost can also have a negative component and thus that quality and cost can move in opposite directions. ${ }^{2}$ Furthermore, while the same health care payers proclaim that they are as motivated as physicians by concern for patients, asserting that reduced cost is simply a byproduct of good management efforts, ${ }^{3}$ in fact their main goal is often cost containment, irrespective of potential drawbacks for patients.

In line with this current belief, the use of cost effectiveness analysis - a method for plotting points on a curve and quantifying the direction of change in quality and cost that occurs with new or alternative modes of diagnosis or treatmenthas been a foundation for health care policy changes, ignoring two essential patient centred concerns:

1. What is best for this patient?

2. How should we distribute limited health care resources fairly?

For the clinician, the method of health care policy making is overtly focused on patient care. The currently accepted method of evidence based medicine-defined as the integration of individual clinical expertise with the best available external evidence and patient's values and expectationshas been accepted as a reasonable and rational way of making decisions about the care of a specific patient. Although this method seems straightforward, the devil is in the details because for most surgical decisions, clear answers or guidance are not available, and the limited scope and ambiguous nature of available evidence introduces ethical concerns about the use of the evidence based framework for "decision making" in the quickly evolving art and science of surgery. These concerns arise from the types of decisions to be made, the type of practice within which they are to be made, and the nature of the evidence available and required for this decision making.

What is clear is that in both managerial and clinical medicine, it is important in applying cost containment choices, cost effectiveness analysis and the evidence based medicine approach to either surgical practice or health care policy, to avoid the twin pitfalls of hastily rejecting the unproven and of creating a straightjacket of protocol driven medicine, thus arresting future progress. In the ultraliberal world surgeons live in, this kind of seemingly protocol driven medicine, when applied to surgical practice, can be rather appropriately described by using market metaphors: now a good surgeon in the managed care paradigm is no longer a surgeon who successfully performs expensive and difficult operations in high risk 
patients. A "good" surgeon performs only profitable operations, balancing the budget of the health maintenance organisation (HMO) to which he belongs, and/or he is a surgeon who is not operating at all. In other words, from a managerial point of view, a good surgeon is one who is not spending the resources of the HMO, whose primary goal in a market environment is reducing cost in order to make a profit, or in a nationalised health care system (which in Europe is functioning more and more as an HMO) saving public money by reducing spending. Surgeons may be resisting EBM because they feel it is often guided by business tenets meant to manage costs, not care.

To put it bluntly: even if there is no rational incompatibility between the components of the trilogy resulting from the fair and efficient containment of costs in health care, the evidence based medicine approach, and the effective care of patients, it is obvious that the interconnection between these three issues is hedged about with the difficulties of defining, with transparency and coherence, what is the reasonable border between macroeconomics (which has to deal with political choices and economical feasibility) and microeconomics (which is confronted at the grass roots level with the ethical and deontological obligations of the clinician). Part of those difficulties are illustrated by the unconscious-but sometimes deliberate-confusion ${ }^{4}$ of the concept of efficiency (which is an economical concept) with the concept of effectiveness (which is a clinical concept).

Nevertheless, we should neither put all the blame on incoherence between the micro and macroeconomics of health care resource allocations, nor confuse deliberately the semantics of buzzwords coined by and for the health care complex. It is precisely here that we have to resort to sound surgical mystique, which does not mean an atmosphere of mystery and veneration investing the art of surgery or any professional skill designed to mystify and impress the lay person. But rather a mystique whose main feature is honest self criticism about: the way surgical results are evaluated; the way surgical innovations are introduced and validated, and about the way clinical and ethical principles are applied more or less strictly to some people or situations than to others, which is exactly how double standards arise.

\section{SURGICAL SELF CRITICISMS The methodology and socioeconomic aspect of the criticisms}

Due to the fact that evidence based medicine relies classically on randomised controlled trials (RCT) as its core source for research design, recent criticism of the quality of surgical research has suggested that surgeons are particularly unwilling to perform randomised controlled trials, and the real proportion of surgical treatments that are based on such trials is not known. With this criticism, surgical clinical standards, which until now have too often enjoyed immunity from scrutiny under the cover of surgical mystique, are no longer beyond question and are now under financial and political pressures to reduce costs: any intervention that cannot be justified as soundly based is potentially liable to restriction or abolition by health care purchasers.

Therefore, it was with some courage that Howes et al decided to tiptoe through such political and financial minefields while carrying out a study of practice in general surgery to determine how much of the activity in the surgical specialty is evidence based. Surgical treatments were placed into one of three categories. Category 1 treatments are supported as effective by a well conducted RCT. Category 2 interventions are those for which no RCT evidence exists, but where there is other evidence sufficient to convince all members of the study group that to conduct a randomised trial would be unnecessary, or, unethical if the comparison were against placebo or no treatment—for example, appendectomy for acute appendicitis $v$ no surgical treatment. Category 3 comprises the remaining treatments, which did not meet the criteria for inclusion in either of the other two categories. In this study, $95 \%$ of the patients received treatment based on satisfactory evidence (categories 1 and 2) and, of these, $24 \%$ of patients received treatments based on RCT evidence (category 1) and $71 \%$ had treatments based on other convincing evidence (category 2). The conclusion was that RCTs are probably not appropriate for many topics in surgical practice because the relevant questions cannot be expressed as choices between defined alternatives of equal weight or value.

Furthering the development of evidence based practices in surgery is not uncomplicated. The practical difficulties of conducting a satisfactory RCT of a surgical procedure are much greater than those encountered with most medical treatments. Standardisation of a complex surgical procedure, unlike the standard administration of most drug regimens, is a major challenge. In fact, if the technique under study is new, the participating surgeons may experience a substantial learning curve before reaching a steady level of performance. Even beyond the stage of the learning curve, performance between surgeons is still likely to vary widely. Furthermore, modification of the surgical technique or the operative strategy in response to particular circumstances in individual patients may appear necessary to the surgeon in a significant proportion of cases. Elimination of such cases from the study would be likely to introduce serious bias as well as increase subject recruitment problems. Last but not least, patient preferences often pose problems in comparisons between a surgical and non-surgical treatment, or between two different operations. Therefore, it is not astonishing that a recent estimate, ${ }^{5}$ based on apparently realistic assumptions, suggested that only $39 \%$ of surgical treatments could be subjected to RCT under ideal conditions.

We can briefly illustrate this by an example about the way new surgical procedures are still actually assessed by the surgical profession: the surgical intervention rates and operative mortality for open cholecystectomy (OC) and laparoscopic cholecystectomy (LC). In fact, the rapid and widespread adoption of laparoscopic cholecystectomy in the early nineties aroused concern about the safety of the new procedure. In addition, one wondered whether the availability of a less invasive approach to cholecystectomy had led to a change in the spectrum of patients undergoing the procedure and in the threshold for performing it. In other words we are back to our two basic questions: what is best for this patient, and how should we distribute health care resources fairly? This example of OC versus LC is also helpful to illustrate the concepts just mentioned, that is to say: standardisation of complex surgical procedures, learning curves before reaching a steady level of performance, and patient preferences.

A non-randomised retrospective study ${ }^{6}$ used the 1985-1992 hospital discharge rate from acute care hospitals in Maryland for open (OC) and laparoscopic (LC) cholecystectomies to identify characteristics of patients undergoing these procedures, and deaths occurring during hospitalisations in which these procedures were performed. The annual rate of cholecystectomy, adjusted for age, rose from 1.69 per 1000 state residents in 1987-1989 to 2.17 per 1000 residents in 1992, an increase of $28 \%(\mathrm{p}<0.001)$. Although the operative mortality associated with laparoscopic cholecystectomy was less than that with open cholecystectomy, and the overall mortality rate for all cholecystectomies declined from $0.84 \%$ in 1989 to $0.56 \%$ in 1992 , there was no significant change in the total number of cholecystectomy related operative deaths because of the increase in the cholecystectomy rate.

So from a clinician's point of view, laparoscopic cholecystectomy would appear to be the best way to treat patients with gallstones. This technique has, however, also produced a shift in health care resources: patients undergoing laparoscopic cholecystectomy tended to be younger, white, to present with 
less serious problems and to have private health insurance or belong to an HMO $(p<0.001)$. The very same kind of shift in health care resource allocation has been found in the countries of Western Europe since the beginning of the blitz of laparoscopic surgery in the 1990s. This example is a clear illustration of the latent conflict that can arise between the macroeconomics of health care resource availability, with its related political willingness towards efficient and fair allocation, and the microeconomics of effective care delivered by first line practitioners fearing for their professional autonomy.

\section{The morbidity aspect of the criticisms}

A major hurdle in evaluating the safety of new surgical techniques is interpreting the significance of the learning curve phenomenon. A prospective analysis of 1518 laparoscopic cholecystectomies from Duke University medical center ${ }^{7}$ showed a total of 82 complications occurring in 78 (5.1\%) of the patients. This is comparable with the reported rates of complications for conventional open cholecystectomy. The rate of the most severe complication-common bile duct or hepatic duct injury-was only $0.5 \%$. The incidence of bile duct injury in the first 13 patients operated on laparoscopically by each surgical group was, however, $2.2 \%$, as compared with $0.1 \%$ for subsequent patients. This is a clear demonstration of the impact of the learning curve when a new surgical procedure or a new surgical approach has yet to be standardised.

Indeed, the severe complication of bile duct injury is the crux of the problem in laparoscopic biliary surgery and this injury does not always manifest itself immediately. It is true that the incidence of ductal injury during LC is not greater than that quoted in previous studies for open cholecystectomy. The short follow up of the available studies (retrospective study average 8.4 weeks follow up, prospective study average 3.7 weeks follow up) should not, however, lead us to overoptimistic conclusions. ${ }^{8}$ It is important to note that only about $10 \%$ of ductal injuries are discovered and operated upon in the first week or so. The vast majority $(70 \%)$ are diagnosed within the first six months and the rest within one year of the operation during which the injury occurred. A small percentage of these injuries become clinically manifest only after many years. Every attempt should be made to decrease the creation of "biliary cripples", and a much longer follow up will be required to evaluate the final incidence of bile duct injuries during LC, another difficulty faced by surgical researchers.

Laparoscopic cholecystectomy is here to stay, but there are many areas which need improvement: operators' skills; training of surgeons who will perform the operation in the future; audits of outcome, and effective peer review mechanisms. Policies to establish the credentials of these additional aspects are important and can influence development and standardisation of the operative procedure. Instrumentation is still evolving, and increasingly challenging cases will be operated upon laparoscopically. If surgeons do not become aware of the shortcomings and double standards in clinical practice and research, and if they do not try to eliminate them, the number of severe complications will increase. In this regard, the most important solution is found in the long history of publishing evidence in national and international peer review journals about those shortcomings and the importance of long term follow up of these patients. Belgian surgeons have already provided a significant amount of quality evidence concerning patients who have benefited from the new videoscopic approaches in many different aspects of the art of surgery, such as biliary surgery or adrenal surgery.

\section{EVIDENCE BASED MEDICINE AND THE DOCTOR PATIENT RELATIONSHIP}

Cost effectiveness analysis and the evidence based medicine approach (EBM) are also here to stay, but their potential to distort the doctor patient relationship is another cause for concern and an additional source of surgical risk. Historically, the physician has been the advocate of the patient. The physician's top priority is to act in the best interest of the patient, acknowledging fully the importance of the patient's own values and perception of his or her health and decisions regarding it. Within this framework, EBM can be useful to the doctor and patient so long as it remains a tool that helps inform their decision making with only the patient's health in mind. However, when EBM is used only to evaluate decisions about resource allocation, there is a risk of EBM becoming a potent force that transforms the physician into an agent of the health service and the patient into a consumer.' At such a system level, the physician becomes a double agent since one person's health care is another person's health cost-a double agent keeping his left eye on patient needs and his right eye on the health care cost containment indicators. Furthermore, when the EBM approach is blindly linked to cost effective/benefit analysis (CEA/CBA) it can lead to the routine rejection of the unproven, and force clinicians into the Procrustean bed of protocol driven medicine. Just as in Greek legend Procrustes mutilated his victims in order to make them fit the length of his bed, so cost analysis in the current market driven climate can lead ineluctably to cost containment and thus to the detriment of the patient.

Evidence based medicine can also lead, however, to a more "rational" provision of diagnostic and therapeutic services, since it provides a focused and more efficient approach to the interpretation of research findings and translates them into clinical options. Consequently, EBM can provide the surgeon with a valuable tool for managing the knowledge base of medicine or surgery. This EBM approach also focuses, however, on average and mean effects and rarely provides clear cut guidance to help the surgeon tailor care and surgical intervention to the individual patient. Even more rarely does EBM provide guidance about how to respond to an individual patient's values, priorities, and cultural needs. The potential role of EBM in resource allocation and health service management must be recognised by surgeons as that of a tool, which can enlighten their decisions and show reasonable trends for health care cost containment. It should not be seen as a standard by which decisions about the allocation of health service resources and the crafting of clinical research agendas and priorities are to be made. In other words, surgeons should not become so bemused by analytical and statistical techniques applied to large amount of data and patients-for the purposes of EBM - that sight of a simple rule is lost: each patient should always be assessed and operated on individually.

\section{PARADIGM OF SURGICAL SCIENCES VERSUS SURGICAL MYSTIQUE?}

A paradigm is made up of general and theoretical assumptions, laws, and techniques. From the philosophical point of view, a paradigm is a mode of viewing the world which underlies the theories and methodology in a particular period of history. For example, the momentous discovery of universal gravitation became the paradigm of successful science. Now we view the world of science in the light of the contemporary Popperian model: experimental results and observations are tests for laws and theories which are conjectures; if a prediction turns out to be correct, the theory from which it followed might still be false. Thus such a model has to do with testing hypotheses and asking the question: "has the hypothesis been confirmed by inductive evidence or rejected as false"? As a consequence, science is systematically collected and arranged knowledge, and research is the attempt to obtain that knowledge.

In this context, EBM appears to provide one answer to the question of what should guide the clinical practice and the distribution of resources: a universal metric through which 
the relative value of alternative investments in health care can be compared. Unfortunately this universal metric has not emerged; measures of mortality, morbidity burden, years of life, years of productive life, and quality adjusted life years (QALYs) have all been proposed, but none are perfect. ${ }^{8}$ The promise of such an approach, with the potential for rationalising the management of health care systems, is seductive to those faced with management decisions. But it is unlikely that universal metrics of the value of health care will ever be developed. Indeed, in some clinical situations, the "right" decision based on reasonable clinical evidence leads to decisions that are judged immoral and contrary to the patient's best interests at an ethical level of decision making. For example, placing patients in restraints can reduce falls among disoriented or elderly patients (a "right" EBM decision), yet the unethical restriction of personal freedom involved has led many institutions to forbid restraints in most circumstances. Even this apparently simple case is not clear! First, which and where is the frontier between the competent and incompetent patient during the early postoperative period? Second, using restraints is often an alibi to hide the fact that the nursing staff has been reduced to a trickle (particularly at night) for financial or economical reasons. Here, we are clearly facing the choices of the kind of society we want to live in. In other words: what amount of money is our society willing to allocate to the improvement-or at least the maintenance-of the quality of modern health care?

Another ethical concern stems from the covert assumption that if a clinical activity cannot be quantified, or has no evidence base, it is of no value. In fact, EBM must never take precedence over sound clinical and ethical decision making by a competent and compassionate physician.

All of this can perhaps illustrate why there is no definitive paradigm in the surgical sciences. Any discovery, regardless of methodology, that benefits the science and practice of surgery is considered by the surgical profession as good surgical research that can eventually lead to sound surgical practice. This is even more so if the discovery produces direct benefit for the patient, considering that quite often surgical decisions cannot be delayed.

Yet the virtually total rejection of controlled trials by surgeons implies a form of schizoid behaviour in the surgical community which requires further examination. In fact, barriers to accepting controlled trials appear to be conceptual and not related to skill or intellect. Those barriers form an interrelated set of misapplied virtues such as faith and activism. Faith implies a voluntary suspension of objectivity (if not reason). Activism implies a compulsion to do something and can arise from the most humanitarian instincts. By extrapolation, the most radical or drastic solution appears to be the most activist; hence, those impressed by activism readily develop faith in reasonable appearing activist solutions. ${ }^{10}{ }^{11}$

\section{SURGICAL INNOVATION, PATIENT VULNERABILITY, AND ETHICS}

The problem is not only a semantic or a philosophical one where innovation in surgery is concerned. Innovation should also invoke morally troublesome issues for the surgical innovator, even though a certain level of innovation is expected in our daily surgical practice when we encounter unanticipated findings: "We couldn't remove the obstructing tumour, but we were able to bypass it". Innovation is highly valued in our societies, but innovation in surgery can lead to unforeseen complications and raises three moral issues. ${ }^{12}$

First, there is the profound trust that vulnerable patients feel toward their surgeon. Even when the surgeon innovates, the patient expects the surgeon to be his advocate for optimal care, not an advocate for innovative research or for some minimalist standard. The risk is that the surgeon will cease to be the conservative guardian, given to using traditional techniques that have been validated by years of experience. This trait is expressed daily in the operating room in many ritualised routines. Members of the operating room team constitute a moral community with strong implicit standards to protect the patient and the surgeon from danger, including dangerous innovations. ${ }^{13}$

Secondly, there is the unbalancing effect of new procedures on traditional safeguards of surgical competence. The term "innovation" has a seductive connotation of added value in our market society. There is even a class of patients who are psychologically disposed to seek innovative treatment because it is the latest and, by sometimes erroneous inference, the best that is available. In surgical practice, however, the patient's preference is not the final word, even though patients behave themselves more and more like consumers. This is the reason why instead of using the word innovation we should use the term "non-validated" to describe the status of newly introduced procedures. ${ }^{12}$ The word non-validated accurately captures the sense of moral hazard that should be attached to the use of newly introduced procedures in vulnerable and trusting patients. "Non-validated" also implies that the expert surgical community still has policies ensuring honesty and fidelity to trust, and that these apply to newly introduced procedures before they are widely accepted and validated.

Thirdly, there is an imperative need for a systematic approach to the evaluation of new surgical procedures and to the accreditation process of training. Under pressure from patients, equipment manufacturers, public media, and insurers-all of which have penetrated the sanctuary of the operating room, bypassing the strong implicit standards of the moral community represented by the operating room teamsurgeons may believe they are required to introduce nonvalidated procedures they have learned in less than ideal forums, such as weekend courses or workshops sponsored by the industry. Often, accreditation of such training and the certification of the skills acquired are problematic. When innovative surgeons return with uncertified skills to introduce nonvalidated treatments in trusting patients, we are denying the basic principles of medical ethics and we have a recipe for clinical disaster.

\section{GENERAL CONCLUSIONS}

Clinically relevant attitudes and/or guidelines issued by a rational EBM approach are attitudes and guidelines that integrate individual clinical expertise with the best available external clinical evidence from systematic research (including metaanalyses). These guidelines should aim to provide an unbiased summary of the evidences base in order to respond to a clinical or health policy question, to identify gaps in the existing clinical research, and to improve the quality of new research. ${ }^{14}$

Sir Miles Irving, director of the British NHS Health Technology Programme, proposed at the annual meeting of the European Society of Surgery in Berlin, ${ }^{15}$ in December 1999, the following list of priorities-the sequence of which is important to consider-that have to be met for a guideline to be considered as "clinically relevant": validity; reliability; charity and compassion; clinical applicability; patient and user involvement; linked to audit; reproducibility; clinical flexibility; scheduled review date; meticulous documentation, and cost effectiveness.

This list of priorities could be useful in transforming the actual reality of surgical double standards. Furthermore, it is somewhat relevant — and reassuring — to note that charity and compassion are in third position and cost effectiveness in last position.

In the world of managed care, surgeons need to develop a health policy agenda that emphasises patient care issuesavailability; freedom of choice, and the patient's best interests-above provider or payer interests. Nevertheless, 
they are also required to enhance their education programmes and the continuing objective assessment of the way surgery is performed and transformed. They should also assume an increased leadership role in developing critical evaluation of non-validated techniques by favouring the development of sound clinical trials and by considering EBM not as a weapon turned against, or targeted at, the surgical profession, but as a valuable tool that may provide some answers to chronically unresolved questions which persist in the art of surgery. ${ }^{16}$

Evidence based medicine is not "cook book medicine", but can provide guidelines and check lists for optimal patient's care. For evidence based medicine to become also consensus based medicine, several steps have to be followed: formulation of precise and answerable clinical questions; searching the literature for current best evidence; assessing the validity, impact, and applicability of the information obtained, and sitting down and discussing in order to confirm that evidence based medicine is really consensus based medicine, which introduces more than a simple nuance between rational and reasonable medical practice. However, to follow this process in order to make choices more coherent will require not only energy and creativity but also time. And as time is money, the health authorities should finally consider the efficient funding of clinical research, which in return could prove to be effective investment in terms of health care for the population and sound choices in term of resource allocation.

If mystique is the atmosphere of mystery and veneration investing some doctrines, arts, professions or people, it can also denote any professional skill or technique designed or able to mystify and impress the lay person. Hence the sequence of the priorities proposed by Miles Irving ${ }^{15}$ could be helpful firstly to stop mystifying the lay patient, and secondly to reverse the current trivial socioeconomic trends in the health care business in which the name of the game is sometimes to avoid caring for sick people by enrolling a disproportionate number of relatively healthy patients-_creaming", or by reducing the contingent of very sick and high risk patients-“dumping". ${ }^{17}$ Above all, such a sequence of priorities could help keep alive the enlightening mystique of the art and profession of surgery: "The secret of patient care is in caring for the patient". ${ }^{18}$

\section{Authors' affiliations}

L A Michel, Surgical Services, Mont-Godinne University Hospital, Université Catholique de Louvain (UCL)-Medical School, Yvoir, Belgium P M Johnson, Department of Internal Medicine, University of Utah, Salt Lake City, USA.

\section{REFERENCES}

1 Howes N, Chagla L, Thorpe $M$, et al. Surgical practice is evidence based. British Journal of Surgery 1997:84:1220-3.

2 Rhodes RS, Rhodes P. Cost-effectiveness analysis in surgery: who will use it and how? Surgery 1998;123:119-20.

3 Bodenheimer T, Casalino L. Executives with white coats - the work and world view of managed-care medical directors (second of two parts). New England Journal of Medicine 1999;341:2029-32.

4 Michel L. Equité, études "coût-efficacité" et evidence based medicine: une illustration en chirurgie. Ethica Clinica 2000;20:14-23.

5 Solomon MJ, McLeod RS. Should we be performing more randomised controlled trials evaluating surgical operations? Surgery 1995:118:459-67.

6 Steiner CA, Bass EB, Talami MA, et al. Surgical rates and operative mortality for open and laparoscopic cholecystectomy in Maryland. New England Journal of Medicine 1994;330:403-8.

7 The Southern Surgeons Club. A prospective analysis of 1518 laparoscopic cholecystectomies. New England Journal of Medicine 1991;324:1073-8

8 Airan M, Appel GB, Coburg AJ, et al. Retrospective and prospective multi-institutional laparoscopic cholecystectomy study organized by the Society of American Gastrointestinal Endoscopic Surgeons (SAGES). Surgical Endoscopy 1992;6:169-76.

9 Culpepper L, Gilbert TT. Evidence and ethics. Lancet 1999;353:829-31.

10 Spodick DH.The surgical mystique and the double standard. American Heart Journal 1973;85:579-83.

11 Michel L. Ethique et repartition des ressources. Revue d'Ethique et Théologie Morale 1993;185:119-35.

12 McKneally MF. Ethical problems in surgery: innovation leading to unforeseen complications. World Journal of Surgery 1998;23:786-8.

13 Michel L. Expériences chirurgicales et innovations médicales. Le Soir 2001 Feb 2: 14.

14 Sheldon TA. Systematic reviews and meta-analyses: the value for surgery. British Journal of Surgery 1999;86:977-8.

15 Irving M. Improving quality in surgery by evidence-based medicine. Keynote lecture of the Annual Congress of the European Society of Surgery (ESS). Berlin, Dec 1999.

16 Michel L, Jamart J, Bradpiece H, et al. Prediction of risk in noncardiac surgery following cardiac surgery. Journal of Thoracic and Cardiovascular Surgery 1990;100:595-605.

17 Kassirer JP, Angell M. Risk adjustment or risk avoidance? New England Journal of Medicine 1998;339:1925-6.

18 Peabody FW. Editorial. Journal of the American Medical Association 1927;88:877. 